3. Zerbib F, des Varannes SB, Roman S, et al. Normal values and day-to-day variability of 24-h ambulatory oesophageal impedance-pH monitoring in a Belgian-French cohort of healthy subjects. Aliment Pharmacol Ther

2005:22:1011-21.

4. Davis RD Jr, Lau CL, Eubanks S, et al. Improved lung allograft function after fundoplication in patients with gastroesophageal reflux disease undergoing lung transplantation. J Thorac Cardiovasc Surg 2003:125:533-42.

5. Bredenoord AJ, Weusten BL, Timmer R, et al. Reproducibility of multichannel intraluminal electrical impedance monitoring of gastroesophageal reflux. Am J Gastroenterol 2005;100:265-9.

\section{Attitudes of patients towards a hospital-based rehabilitation service for obesity hypoventilation syndrome}

Obesity hypoventilation syndrome (OHS) is characterised by a body mass index of $>30 \mathrm{~kg} / \mathrm{m}^{2}$, with either diurnal or nocturna hypoventilation in the absence of any other explanation for this. ${ }^{1}$ If left untreated, OHS can be associated with significant morbidity and mortality. Currently, the mainstay of treatment for $\mathrm{OHS}$ is non-invasive ventilation. Pulmonary rehabilitation (PR) is an established form of treatment for patients with chronic obstructive pulmonary disease (COPD). ${ }^{2}$ PR is increasingly offered to patients with various other chronic respiratory diseases, and a similar programme with particular emphasis on obesity can reasonably be expected to benefit patients with OHS.

At Papworth hospital, we have been interested in developing a multidisciplinary rehabilitation programme for patients with $\mathrm{OHS}$ and performed a questionnaire-based survey investigating the likelihood of patients participating in such a programme.

Patients were identified from the hospital database. A letter outlining the proposed rehabilitation programme (visiting the hospital twice a week for 8-12 weeks for a series of sessions involving supervised exercise, dietary advice and educational classes) and a short anonymous questionnaire were sent to 96 patients with OHS. The questionnaire required the patients to specify how likely they would be to attend the programme, if offered, and the reasons that might prevent them from attending. The patients were also asked if they would be more likely to attend the rehabilitation if travel was arranged and, if so, whether they would prefer the hospital to arrange transport or to be reimbursed for their own arrangements. The questionnaire included a section for any comments patients had.

Forty-six (48\%) patients returned the questionnaire. Of these responses, 14 (30\%) patients would either be "very likely" or "likely" to attend the rehabilitation programme, with 20 (43\%) patients being "not at all" likely to attend. Travelling to the hospital was found to be the most significant concern amongst the group, with 27
(59\%) patients saying the distance or travelling involved would prevent them from attending the programme. Out of these 27 patients, $11(41 \%)$ would still not attend if their travel was arranged for them. Inconvenience $(9 \%)$ and lack of spare time $(13 \%)$ were also cited as reasons that prevented participation in the programme. Travelling was mentioned most frequently $(37 \%)$ as well as patients reporting that the proposed rehabilitation programme would conflict with existing commitments such as work or child care $(24 \%)$, that it would be more convenient if carried out in a local healthcare facility (13\%) and that they needed more information in order to decide whether to attend (11\%).

Given that the most common concern amongst patients was the travel required to attend the programme, one might predict that offering to help patients with their travel arrangements would increase the likelihood of attending rehabilitation. However, despite offering two options of travel assistancearranged by the hospital or reimbursement for their own arrangements - $41 \%$ of patients would still not attend.

This survey suggests that patients with $\mathrm{OHS}$ are poorly motivated to attend a rehabilitation programme. Providing more information about the benefits of exercise and diet-based interventions and a local service with adequate travel arrangements may increase the attendance at such programmes.

\section{K E Jordan, M Ali, J M Shneerson}

Papworth Hospital NHS Foundation Trust, Cambridge, UK

Correspondence to: Dr J M Shneerson, Papworth Hospital NHS Foundation Trust, Cambridge CB23 3RE, UK; john.shneerson@papworth.nhs.uk

Competing interests: None.

Provenance and peer review: Not commissioned; not externally peer reviewed.

Accepted 7 June 2009

Thorax 2009;64:1007. doi:10.1136/thx.2009.120808

\section{REFERENCES}

1. Mokhlesi B, Tulaimat A. Recent advances in obesity hypoventilation syndrome. Chest 2007;132:1322-6.

2. Casabury R, ZuWallack R. Pulmonary rehabilitation for management of chronic obstructive pulmonary disease. N Engl J Med 2009:360:1329.

\section{Merkel cell polyomavirus is prevalent in a subset of small cell lung cancer: a study of 31 patients}

Merkel cell carcinoma (MCC) and small cell lung cancer (SCLC) share quite distinct similarities such as almost undistinguishable histological presentation (fig 1a,b) and highly aggressive biological behaviour with high rates of metastasis and poor survival rates. ${ }^{12}$ While tobacco smoking and genetic susceptibility have been identified as risk factors for $\mathrm{SCLC}^{34}$ sun exposure and immunosuppression are the main risk factors for MCC. ${ }^{1}$ In January 2008, a new virus, called Merkel cell polyomavirus (MCPyV), was desribed by Feng et al as a likely causative agent of MCC, proving monoclonal MCPyV-genome integration in 8 of 10 MCCs. ${ }^{5}$ Since then these findings have been reproduced by several groups. ${ }^{6}$

Because SCLC and MCC share obvious similarities in histological presentation, Wetzels et al recently published the first report looking for $\mathrm{MCPyV}$ prevalence in SCLC. ${ }^{8}$ They investigated a small cohort of 10 patients with SCLC, finding no prevalence for MCPyV. We performed a molecular pathology study in a relatively large cohort of 31 patients (36 samples) analysing the presence of MCPyV DNA by PCR and chemiluminescence Southern blot hybridization of PCR products. These data are the first to test the findings of Wetzels and colleagues.

Based on the DNA sequences published by Feng et al, we designed two sets of primers in order to test the formalin-fixed and paraffinembedded (FFPE) tissues for the presence of specific MCPyV DNA. After DNA extraction from FFPE tissues, PCR amplification using these primer combinations resulted in a $138 \mathrm{bp}$ product (MCV138 forward: 5'-GGTTAGAGATGCTGGAAATGACC-3'; reverse: 5'-CAAATAAGCAGCAGTACCAGGC-3') and a $191 \mathrm{bp}$ product (MCV191 forward, 5'-CCACTTTATTATCTTAGCCCAT-3'; and reverse, 5'-TCCTTTTGGCTAGAACAGTGTC-3') targeting the large and small $\mathrm{T}$-antigen region of MCPyV (isolate MCC-Mpt-LS2) Results were confirmed by chemilumeniscence Southern blotting with specific probes for MCV138 and MCV191, omitting primer sequences and carrying a 5 digoxigenin label (DIG-MCV138 forward, 5'-GTAAGAAGTTTAAGAAGCACCTAG-3'; and DIG-MCV191 forward, 5'-GATCTCGCCTCAAACCTCACAAG-3'). DNA quality was confirmed by $\beta$-globin PCR using the GH20 (5'-GAAGAGCCAAGGACAGGTAC-3') and PCO4 (5'-CAACTTCATCCACGTTCACC-3') primer set.

Of the 36 SCLC specimens, 35 revealed a $\beta$ globin PCR product. These 35 specimens were from 30 patients ( 13 women and 17 men, clinically not differentiated in limited and extensive disease; 2 patients with three samples each and 1 patient with two samples). Their mean (SD) age was 67.9 (8.6) years. MCPyV sequences were detected in 2 of 30 patients $(7.5 \%)$, specifically in three of 35 samples (two of these three samples are from 1 patient, both positive for MCV138 (fig 1c,d) and the remaining sample was positive for MCV191). The PCR-negative controls, containing all other PCR components and water instead of DNA, were constantly negative in all experiments.

Based on the data presented here, it is unlikely that MCPyV plays a significant pathogenetic role in SCLC; nevertheless a supplemental role as cofactor in the pathogenesis of SCLC cannot be ruled out 